\title{
MOP_Algorithmic Modality Analysis for Parabolic Group Actions
}

\author{
UIf Jürgens and Gerhard Röhrle
}

\section{CONTENTS}

1. Introduction

2. Notation and Preliminaries

3. Some Applications of MOP

4. The MOP Program

Acknowledgments

References
2000 AMS Subject Classification: Primary 20G15; Secondary 17B45

Keywords: Linear algebraic groups, Lie algebras, modality of parabolic groups.
Let $G$ be a simple algebraic group and $P$ a parabolic subgroup of $G$. The group $P$ acts on the Lie algebra $\mathfrak{p}_{u}$ of its unipotent radical $P_{u}$ via the adjoint action. The modality of this action, $\bmod \left(P: \mathfrak{p}_{u}\right)$, is the maximal number of parameters upon which a family of $P$-orbits on $\mathfrak{p}_{u}$ depends. More generally, we also consider the modality of the action of $P$ on an invariant subspace $\mathfrak{n}$ of $\mathfrak{p}_{u}$, that is mod $(P: \mathfrak{n})$. In this note we describe an algorithmic procedure, called MOP, which allows one to determine upper bounds for $\bmod (P: \mathfrak{n})$.

The classification of the parabolic subgroups $P$ of exceptional groups with a finite number of orbits on $\mathfrak{p}_{u}$ was achieved with the aid of MOP. We describe the results of this classification in detail in this paper. In view of the results from [Hille and Röhrle 99], this completes the classification of parabolic subgroups of all reductive algebraic groups with this finiteness property.

Besides this result we present other applications of MOP, and illustrate an example.

\section{INTRODUCTION}

Throughout, $G$ is a simple algebraic group over an algebraically closed field $K$ and $P$ is a parabolic subgroup of $G$. The group $P$ acts on its unipotent radical $P_{u}$ via conjugation and on $\mathfrak{p}_{u}$, the Lie algebra of $P_{u}$, via the adjoint action. The modality of the action of $P$ on $\mathfrak{p}_{u}$, denoted by $\bmod \left(P: \mathfrak{p}_{u}\right)$, is the maximal number of parameters upon which a family of $P$-orbits on $\mathfrak{p}_{u}$ depends. More generally, we also want to study the modality of the action of $P$ on an invariant linear subspace $\mathfrak{n}$ of $\mathfrak{p}_{u}$, that is $\bmod (P: \mathfrak{n})$. The modality of $P$ is defined as $\bmod P:=\bmod \left(P: \mathfrak{p}_{u}\right)$; see Section 2 for a precise definition and [Popov and Röhrle] for some additional references concerning this notion. Observe that $\bmod (P: \mathfrak{n})$ is zero precisely when $P$ operates on $\mathfrak{n}$ with a finite number of orbits.

In this paper we describe the modality algorithm MOP (Modality Of Parabolics) which is designed to compute

(c) A K Peters, Ltd. 1058-6458/2001 \$0.50 per page Experimental Mathematics 11:1, page 57 
upper bounds for $\bmod (P: \mathfrak{n})$. In [Popov and Röhrle] the general problem was posed to determine each parabolic subgroup $P$ of $G$ with $\bmod P=0$. After the cases for Borel and semisimple rank one parabolic subgroups were classified in [Kashin 90] and [Popov and Röhrle], respectively, all modality zero parabolic subgroups of classical groups were classified in [Hille and Röhrle 99]. One of the aims of this paper is to extend these results to all of the exceptional groups with the aid of MOP.

Apart from these results we indicate other applications of MOP. For instance, we determine $\bmod P$ for some parabolics in $E_{6}$ by combining lower bounds for $\bmod P$ from [Röhrle 97] with upper bounds obtained by MOP. The algorithm is implemented as a share package in the computer algebra system GAP [GAP 97]. MOP generalizes the algorithm outlined in [Bürgstein and Hesselink 87] which was designed to analyze the orbit structure of a Borel subgroup $B$ for the adjoint and coadjoint actions on $\mathfrak{b}_{u}$ and on $\mathfrak{b}_{u}^{*}$. MOP only applies in the case when $G$ is simply laced. For details on usage and technical aspects the reader should consult the MOP manual [Jürgens and Röhrle 01] (available from the MOP home page http://www.mat.bham.ac.uk/G.E.Roehrle/MOP.html).

The basic machinery for investigating the modality of parabolic subgroups of reductive groups was introduced in [Popov and Röhrle]. There are several recent articles related to this subject, such as [Brüstle and Hille 00], [Hille and Röhrle 99], [Jürgens and Röhrle 98], [Popov 97], and [Röhrle 99].

Our general reference for algebraic groups is Borel's book [Borel 91] and for information on root systems we refer the reader to Bourbaki [Bourbaki 75]. The simple roots in a base of a root system of $G$ are indexed in accordance with [Bourbaki 75, Planches I - IX].

\section{NOTATION AND PRELIMINARIES}

Suppose that the connected algebraic group $R$ acts morphically on the algebraic variety $X$. For $x$ in $X$ the $R$ orbit in $X$ through $x$ is denoted by $R \cdot x$. The modality of the action of $R$ on $X$ is defined as

$$
\bmod (R: X):=\max _{Z} \min _{z \in Z} \operatorname{codim}_{Z} R \cdot z,
$$

where $Z$ runs through all irreducible $R$-invariant subvarieties of $X$. In case $X$ is an irreducible variety let $K(X)^{R}$ denote the field of $R$-invariant rational functions on $X$. By a result of Rosenlicht $\min _{x \in X} \operatorname{codim}_{X} R \cdot x=$ $\operatorname{trdeg} K(X)^{R}$, for instance, see [Popov and Vinberg 94, 2.3]. Therefore, $\bmod (R: X)$ measures the maximal number of parameters upon which a family of $R$-orbits on $X$ depends. The modality of the action of $R$ on $X$ is zero precisely when $R$ admits only a finite number of orbits on $X$, see also [Popov and Vinberg 94, 5.2].

We denote the Lie algebra of $G$ by Lie $G$ or by $\mathfrak{g}$; likewise for subgroups. The Lie algebra of $P_{u}$ is denoted by $\mathfrak{p}_{u}$. Let $T$ be a maximal torus in $G$ and $\Psi$ the set of roots of $G$ with respect to $T$. Fix a Borel subgroup $B$ of $G$ containing $T$ and let $\Pi$ be the set of simple roots of $\Psi$ defined by $B$, then $\Psi^{+}=\Psi(B)$ is the set of positive roots of $G$.

We may assume that every parabolic subgroup of $G$ under consideration contains $B$, i.e. is standard. For a subset $J$ of $\Pi$ we denote by $P_{J}$ the standard parabolic subgroup corresponding to $J$ such that $P_{\varnothing}=B$. Further, $\ell\left(P_{u}\right)$ denotes the length of the descending central series of $P_{u}$, that is its class of nilpotency. We denote the Weyl group of some Levi subgroup of $P$ by $W_{P}$. By saying that $P$ is of a particular type, we mean the Dynkin type of a Levi subgroup of $P$.

A prime dividing one of the structure constants of the Chevalley commutator relations for $G$ is called a very bad prime for $G$.

Let $\beta \in \Psi^{+}$. Write $\beta=\sum_{\alpha \in \Pi} c_{\alpha}(\beta) \alpha$, with $c_{\alpha}(\beta) \in$ $\mathbb{Z}_{0}^{+}$for each $\alpha \in \Pi$. For $J$ a subset of $\Pi$, we call $\sum_{\alpha \in \Pi \backslash J} c_{\alpha}(\beta)$ the $J$-height of $\beta$; cf. [Azad et al. 90]. For $J=\varnothing$ this is the usual height function.

If char $K$ is not a very bad prime for $G$ and $P=P_{J}$, then $\ell\left(P_{u}\right)$ is just the $J$-height of the highest root in $\Psi$, [Azad et al. 90, Lem. 4].

Let $H$ be a closed connected subgroup of $G$ normalized by $T$ (that is $H$ is a regular subgroup of $G$, cf. [Dynkin $57]$ ); likewise for subalgebras of $\mathfrak{g}$. In that case the root spaces of $\mathfrak{h}$ relative to $T$ are also root spaces of $\mathfrak{g}$ relative to $T$, and the set of roots of $H$ with respect to $T, \Psi(H)$, is a subset of $\Psi$.

If char $K$ is not a very bad prime for $G$, then $\Psi(H)$ is closed under addition in $\Psi$. Furthermore, if $H$ is reductive and regular, then $\Psi(H)$ is a semisimple subsystem of $\Psi$. For a root $\alpha$ of $G$ we denote by $U_{\alpha}$ the corresponding one-parameter unipotent subgroup of $G$. For every root $\alpha$ we choose a generator $x_{\alpha}$ of the corresponding root space Lie $U_{\alpha}=\mathfrak{g}_{\alpha}$ of $\mathfrak{g}$.

The support of a subset $S$ of $\mathfrak{b}_{u}$, denoted by $\operatorname{supp} S$, is the set of all roots $\alpha$ such that the restriction to $S$ of the projection from $\mathfrak{b}_{u}$ onto $\mathfrak{g}_{\alpha}$ is non-trivial.

By a Levi subgroup of a reductive group $G$ we simply mean a Levi subgroup of some parabolic subgroup of $G$.

We require some basic facts concerning the modality of parabolic groups; the first one is elementary (cf. [Popov and Röhrle, Lem. 4.3], or [Röhrle 96, Lem. 2.8]): 
Lemma 2.1. Let $Q \subseteq P$ be parabolic subgroups of $G$. Then $\bmod P \leq \bmod Q$.

This follows readily from the definition, since $\mathfrak{p}_{u} \subseteq \mathfrak{q}_{u}$ and any irreducible $P$-invariant subvariety of $\mathfrak{p}_{u}$ is also $Q$-invariant.

For an automorphism $\Theta$ of $G$ we denote the set of fixed points by $G^{\Theta}$, likewise for $\Theta$-stable subgroups of $G$. We recall [Röhrle 99, Thm. 1.1] (cf. [Popov and Röhrle, Cor. $2.8])$ :

Lemma 2.2. Suppose that $\Theta$ is a semisimple automorphism of $G$ and that $P$ is $\Theta$-stable. Then $\bmod P^{\Theta} \leq$ $\bmod P$.

We require Theorem 1.2 from [Röhrle 99], see also [Popov 97, Thm. 4], [Popov and Röhrle, Rem. 2.14]:

Lemma 2.3. Suppose that char $K$ is zero or a good prime for $G$. Let $H$ be a closed reductive subgroup of $G$ normalized by $T$. Set $Q:=P \cap H$. Then $\bmod Q \leq \bmod P$.

Remark 2.4. In the special case of Lemma 2.3 when $H$ is a Levi subgroup of $G$ normalized by $T$ or the derived subgroup thereof the statement of Lemma 2.3 is valid without any characteristic restrictions [Röhrle 99, Cor. $3.10]$.

For $l \in \mathbb{N}_{0}$ let $\mathfrak{p}_{u}^{(l)}$ denote the $l$-th term of the lower central series of $\mathfrak{p}_{u}$.

Remark 2.5. If char $K$ is not a very bad prime for $G$, then $\mathfrak{p}_{u}^{(l-1)} / \mathfrak{p}_{u}^{(l)} \cong \oplus \mathfrak{g}_{\alpha}$, where the sum is taken over all those roots $\alpha$ in $\Psi$ of $J$-height $l \in \mathbb{N}$, see [Azad et al. 90, Lem. 4].

Observe that in the context of our algorithm this hypothesis on char $K$ is always fulfilled, since MOP only applies when $G$ is simply laced and then the structure constants of the commutator relations are \pm 1 .

We need a special case of a general theorem due to R.W. Richardson [Richardson 85, Thm. E].

Lemma 2.6. Let $P=L P_{u} \subset G$ be a parabolic subgroup of $G$. Then $L$ operates on the quotient $\mathfrak{p}_{u}^{(l)} / \mathfrak{p}_{u}^{(l+1)}$ with a finite number of orbits for each $l \geq 0$.

Remark 2.7. If char $K$ is zero or a good prime for $G$, then $\bmod \left(P: P_{u}\right)=\bmod \left(P: \mathfrak{p}_{u}\right)$ thanks to [Röhrle 99 , Thm. 1.3]. Thus we obtain similar results for the action of $P$ on $P_{u}$.
The statements in Lemmas 2.1 and 2.3 (and the one in Remark 2.7) also apply if we replace $\mathfrak{p}_{u}$ by some $P$-invariant linear subspace $\mathfrak{n}$ of $\mathfrak{p}_{u}$, see [Röhrle 99, Rem. 3.13].

\section{SOME APPLICATIONS OF MOP}

The original motivation for developing MOP was to determine modality zero parabolics in exceptional groups, i.e., ones with a finite number of orbits on the Lie algebra of the unipotent radical. A complete description of all these instances was achieved with the aid of MOP. This is the chief result of this paper:

Theorem 3.1. Suppose $G$ is of exceptional type and that char $K$ is either zero or a good prime for $G$. Let $P \subseteq G$ be parabolic. Then $\bmod P=0$ if and only if one of the following holds:

(i) $\ell\left(P_{u}\right) \leq 4$;

(ii) $G$ is of type $E_{6}, \ell\left(P_{u}\right)=5$, and $P$ is of type $A_{1}^{2} A_{2}$ or $A_{3}$;

(iii) $G$ is of type $E_{7}, \ell\left(P_{u}\right)=5$, and $P$ is of type $A_{1} A_{4}$.

Combined with the analogous result for classical groups [Hille and Röhrle 99], Theorem 3.1 completes the classification of parabolic subgroups $P$ of reductive groups with a finite number of orbits on $\mathfrak{p}_{u}$; this problem was first posed in [Popov and Röhrle].

Whenever char $K$ is zero or a good prime for $G$, we have $\bmod \left(P: P_{u}\right)=\bmod \left(P: \mathfrak{p}_{u}\right)$, thanks to [Röhrle 99, Thm. 1.3]; thus we obtain the finiteness statement of Theorem 3.1 also for the action of $P$ on $P_{u}$.

For the proof of Theorem 3.1 we need to recall the exceptional cases from [Röhrle 96, Thm. 3.1] and [Jürgens and Röhrle 98, Lem. 3.13] and combine them in our next result:

Proposition 3.2. Suppose $G$ is of exceptional type and char $K$ is either zero or a good prime for $G$. Let $P$ be a parabolic subgroup of $G$. Then $P$ acts on $\mathfrak{p}_{u}$ with an infinite number of orbits provided one of the following holds:

(i) $G$ is of type $E_{8}, F_{4}$, or $G_{2}$ and $\ell\left(P_{u}\right) \geq 5$;

(ii) $G$ is of type $E_{6}$ or $E_{7}$ and $\ell\left(P_{u}\right) \geq 6$;

(iii) $G$ is of type $E_{6}, \ell\left(P_{u}\right)=5$, and $P$ is not of type $A_{1}^{2} A_{2}$ or $A_{3}$; 
(iv) $G$ is of type $E_{7}, \ell\left(P_{u}\right)=5$, and $P$ is not of type $A_{1} A_{4}$.

Proof of Theorem 3.1: It follows from Proposition 3.2 that $\bmod P>0$ provided none of the conditions of Theorem 3.1 is satisfied.

In each of the cases of Theorem 3.1 when the Dynkin diagram of $G$ is simply laced the desired finiteness statements was obtained directly using the MOP algorithm. The classification of modality zero parabolics in $G_{2}$ already follows from [Bürgstein and Hesselink 87, Table 2] and [Popov and Röhrle, Thm. 4.2].

Thus, only the instances of $F_{4}$ remain. Let $G$ be of type $E_{6}$ and let $\tau$ be the graph automorphism of $G$ of order 2. The fixed point subgroup $G^{\tau}$ is of type $F_{4}$. Let $Q$ be a parabolic subgroup of $G^{\tau}$. Then, $\bmod Q>0$ provided $\ell\left(Q_{u}\right) \geq 5$, by Proposition 3.2(i). In order to show the converse it suffices to prove that $\bmod Q=0$ provided $Q$ is minimal with respect to satisfying $\ell\left(Q_{u}\right) \leq 4$, by Lemma 2.1. This leads to the three instances when $Q$ is of type $B_{2}, A_{1} \widetilde{A}_{2}$, or $\widetilde{A}_{1} A_{2}$, where $\widetilde{A}_{i}$ represents a subsystem of type $A_{i}$ consisting of short roots. Each such $Q$ can be realized as the $\tau$-fixed point subgroup of a parabolic subgroup $P$ of $G$; see Figure 1. Each occurring $P$ satisfies $\ell\left(P_{u}\right) \leq 4$ and thus $\bmod P=0$ by the finiteness result for $E_{6}$. The desired result for $F_{4}$ then follows by Lemma 2.2 , as $\tau$ is semisimple ( $\operatorname{char} K \neq 2$ ).

Figure 1 presents the crucial $F_{4}$ cases from the proof of Theorem 3.1. The solid nodes indicate the Levi subgroup of $P$ and $P^{\tau}$, respectively.

Owing to the Chevalley commutator relations, $\ell\left(P_{u}\right)$ is readily determined to be the sum of the coefficients of the simple roots $\alpha$ in the highest root of $\Psi$ such that $\mathfrak{g}_{\alpha} \subseteq \mathfrak{p}_{u}$, as indicated in Figure 1 .

We proceed with two a posteriori consequences of the classification of modality zero parabolic groups.

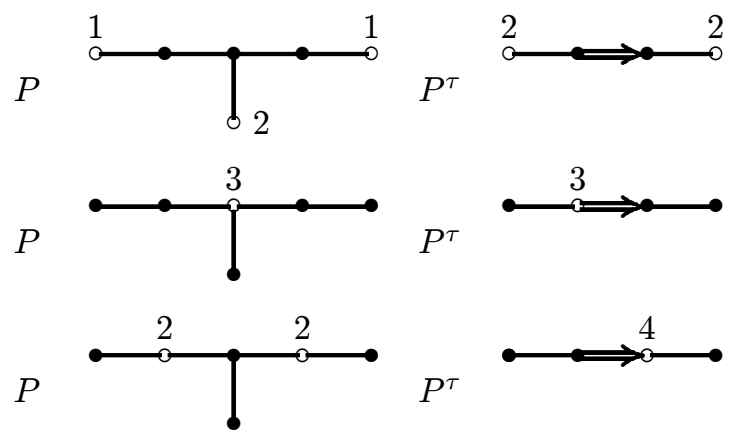

FIGURE 1. The crucial $F_{4}$ cases from Theorem 3.1.
Remark 3.3. Suppose $P$ and $Q$ are associated parabolic subgroups of $G$. Then $\bmod P=0$ if and only if $\bmod Q=$ 0. This follows from [Hille and Röhrle 99, Thm. 1.1] and Theorem 3.1 using the classification of the conjugacy classes of parabolic subsystems of $\Psi$ (cf. [Bala and Carter 76, Prop. 6.3], [Dynkin 57, Thm. 5.4]).

This supports the conjecture that more generally $\bmod P=\bmod Q$ whenever $P$ and $Q$ are associated parabolic subgroups of $G$.

Remark 3.4. Suppose that $\bmod P>0$. Then there exists a connected simple regular subgroup $H$ of $G$ such that the parabolic $Q:=H \cap P$ of $H$ is the standard Borel subgroup of $H$ and $\bmod P \geq \bmod Q>0$. More specifically, $H$ can always be chosen to be of type $A_{5}, B_{3}, C_{3}, D_{4}$, or $G_{2}$. This is a consequence of the inductive construction of all the cases when $P$ is of positive modality, see [Röhrle 96, Thm. 3.1], [Jürgens and Röhrle 98, Lem. 3.13], and [Hille and Röhrle 99, Lem. 2.3].

Some of the cases of Theorem 3.1 are also valid for certain bad primes for $G$; see Section 4.9.

The algorithm can be applied more widely to determine parabolic subgroups of higher modality. For instance, for Borel subgroups $B$ of simple groups of small rank $\bmod B$ can be determined by combining lower bounds for $\bmod B$ from [Röhrle 97] with upper bounds calculated by MOP, extending results from [Jürgens and Röhrle 98]; the latter are based on a precursor of MOP.

More specifically, as an application we calculate explicit upper bounds for the modality of all parabolics in case $G$ is of type $E_{6}$ :

\begin{tabular}{|l|ccccccc|}
\hline Type of $P$ & Borel & $A_{1}$ & $A_{1}^{2}$ & $A_{1}^{3}$ & $A_{2}$ & $A_{1} A_{2}$ & $A_{2}^{2}$ \\
$\bmod P \leq$ & 5 & 4 & 3 & 2 & 2 & 1 & 1 \\
\hline
\end{tabular}

TABLE 1. Upper bounds for $\bmod P$ in $E_{6}$.

In all other instances we have $\bmod P=0$. For $P=P_{J}$ in Table 1 it follows from a construction in [Röhrle 97] that the given values are in fact also lower bounds for $\bmod P$ whenever one of the following holds: $P$ is of type $A_{1} A_{2}$ or $A_{2}^{2}$, or $J$ does not contain the triality simple root. That is, in these instances $\bmod P$ equals the value shown.

Besides computing upper bounds for $\bmod \left(P: \mathfrak{p}_{u}\right)$, we can specify an arbitrary invariant linear subspace $\mathfrak{n}$ of $\mathfrak{p}_{u}$ and calculate an upper bound for $\bmod (P: \mathfrak{n})$. Using a particular feature of MOP we can specify such a subspace $\mathfrak{n}$ and can analyze the $P$-orbits in $\mathfrak{n}$. Remark 3.6 
is such an instance. (In fact $\mathfrak{n}$ does not have to be $P$ invariant, we can simply specify any subspace $\mathfrak{n}$ and then we can analyze the modality of the action of $P$ on the $P$ saturation $P \cdot \mathfrak{n}$ of $\mathfrak{n}$.) For details concerning this feature, see [Jürgens and Röhrle 01].

We discuss a further consequence of the classification results from [Hille and Röhrle 99] and Theorem 3.1.

Corollary 3.5. Suppose $P$ is a non-maximal parabolic subgroup of $G$ and $\bmod P>0$. Then there exists a proper $P$-invariant subspace $\mathfrak{n}$ of $\mathfrak{p}_{u}$ such that $\bmod (P: \mathfrak{n})>0$.

Proof: This follows from an analysis of the inductive construction of all the instances when $\bmod P>0$. More precisely, the statement follows from the classification results in [Hille and Röhrle 99] and Theorem 3.1, the argument of the proof of [Röhrle 96, Thm. 6.3], together with [Hille and Röhrle 99, Lem. 3.2] and [Jürgens and Röhrle 98, Lem. 3.13].

The following $E_{8}$ example illustrates that the statement of Corollary 3.5 is false if the non-maximality condition on $P$ is relaxed.

Remark 3.6. Suppose $G$ is of type $E_{8}$ and $P$ is conjugate to $P_{J}$, where $J=\Pi \backslash\left\{\sigma_{5}\right\}$. It was shown in [Röhrle 96] that $\bmod P>0$. Since $P$ is a maximal parabolic subgroup of $G$, the various members of the descending central series of $\mathfrak{p}_{u}$ are the only $P$-invariant linear subspaces of $\mathfrak{p}_{u}$. Using MOP one can show that $\bmod \left(P: \mathfrak{p}_{u}^{\prime}\right)=0$. In particular, $\bmod (P: \mathfrak{n})=0$ for every proper $P$-invariant subspace $\mathfrak{n}$ of $\mathfrak{p}_{u}$.

As a consequence, $P$ admits a dense orbit on $\mathfrak{p}_{u}^{(i)}$ for each $i \geq 1$. By Richardson's Dense Orbit Theorem [Richardson 74] $P$ also has a dense orbit on $\mathfrak{p}_{u}$ itself. Consequently, every $P$-invariant linear subspace of $\mathfrak{p}_{u}$ is a prehomogeneous vector space for $P$, but nevertheless, $\bmod P>0$.

\section{THE MOP PROGRAM}

In this chapter we give a description of the modality algorithm MOP and discuss some of its features. For further details on usage we refer to the MOP manual [Jürgens and Röhrle 01]. Throughout this chapter, the notation of the previous sections is in force. Let $P=P_{J}$ be a standard parabolic subgroup of $G$ for some $J \subset \Pi$.

\subsection{MOP}

As mentioned above, our algorithm is implemented in the computer algebra system GAP. The advantage of using GAP for our purposes is that firstly it comes with a useful package called CHEVIE for calculations involving Weyl groups and root systems [Geck et al. 96], and secondly it provides convenient data structures, so-called records, to handle objects which depend on a variety of different parameters specified in record fields. For further information concerning commands in GAP we refer the reader to the GAP and CHEVIE manuals [GAP 97], [Geck et al. 96]. The source file containing the program and data is built up in a similar fashion as the files in GAP. Loading the MOP package is achieved by the following command:

gap> RequirePackage ("mop") ;

The fact that CHEVIE is not available as of yet for GAP4, limits MOP to GAP Version 3.4.4.

\subsection{Strings}

Instead of working with individual orbits of $P$ on $\mathfrak{p}_{u}$, we simultaneously consider all $P$-orbits passing through particular kinds of affine subvarieties $S$ of $\mathfrak{p}_{u}$ of the form $S=\sum K x_{\beta}+\sum M x_{\gamma}$, where $M=K \backslash\{0\}$, and the two sums are taken over disjoint subsets of $\Psi\left(\mathfrak{p}_{u}\right)$, of which one may possibly be empty (that is $S \cong M^{a} K^{b}$, where $a, b \in \mathbb{N}_{0}$ ). In particular, $S$ is a locally closed affine subvariety of $\mathfrak{p}_{u}$. Such a subvariety of $\mathfrak{p}_{u}$ is called a string or a string of $\mathfrak{p}_{u}$. The support of $S$ is the set of roots in $\Psi\left(\mathfrak{p}_{u}\right)$ whose coefficient is either $M$ or $K$.

With respect to a total ordering of $\Psi^{+}$, such a subvariety $S$ can be represented symbolically by a sequence of symbols " 0 ", " $M$ ", and " $K$ ", where a "0" indicates that the corresponding root, say $\beta$, is not in the support of $S$, while $x_{\beta}$ has a nonzero coefficient in case of the label " $M$ " and an arbitrary one if " $K$ " occurs, cf. [Bürgstein and Hesselink 87], [Popov and Röhrle, §6], and [Jürgens and Röhrle 98]. This explains the origin of this terminology. For example, for $A_{3}$ the usual ordering of positive roots is $\alpha_{4}=\alpha_{1}+\alpha_{2}, \alpha_{5}=\alpha_{2}+\alpha_{3}$, and $\alpha_{6}=\alpha_{1}+\alpha_{2}+\alpha_{3}$. Therefore, the subvariety $M x_{\alpha_{1}}+M x_{\alpha_{1}+\alpha_{2}}+K x_{\alpha_{2}+\alpha_{3}}$ of $\mathfrak{b}_{u}$ is represented by the string " $M 00 M K 0$ ".

In MOP a string $S$ is a record with two record fields $\mathrm{m}$ and $\mathrm{k}$, which are Boolean lists for the positions of $S$ labeled with $M$ or $K$, respectively. The print function for the record (string) $S$ returns the root numbers of the support of $S$.

The concept behind the program is straightforward. Suppose we aim to show that $\bmod P \leq m$, where $m \in \mathbb{N}$. The basic objective is to construct a finite set of strings $S$ of $\mathfrak{p}_{u}$ with the property that firstly, every $P$-orbit in $\mathfrak{p}_{u}$ has a representative in some $S$ in this list, and secondly, $\bmod (P: P \cdot S) \leq m$ for each such $S$. The de- 
sired inequality $\bmod P \leq m$ then follows, since $\bmod P=$ $\max _{S} \bmod (P: P \cdot S)$, where the maximum is taken over all strings $S$ in our finite collection. MOP proceeds to construct such a finite set of strings by an intricate iteration process of various splitting and elimination operations discussed below.

\subsection{The Stack}

A finite collection $\mathcal{S}$ of such subvarieties $S$ of $\mathfrak{p}_{u}$ satisfying the first property that every $P$-orbit on $\mathfrak{p}_{u}$ is represented by some string $S$, is called a stack of $P$ or a stack of strings of $P$. In our analysis, we initially start out with the stack $\mathcal{S}$ consisting only of the string $\mathfrak{p}_{u}$ itself. By setting an optional parameter when calling the MOP function Modality it is possible to initialize the stack with another string $S$ in order to compute $\bmod (P: P \cdot S)$, see [Jürgens and Röhrle 01].

\subsection{Operations on Strings}

We perform two operations on strings in the stack $\mathcal{S}$ which preserve the property that each $P$-orbit in $\mathfrak{p}_{u}$ passes through some $S$ in $\mathcal{S}$. These operations are aimed at reducing the support of the strings in the stack $\mathcal{S}$, while preserving this property of $\mathcal{S}$ at the same time. The term "stack" reflects the fact that MOP keeps the strings in the well-known data structure of the same name.

4.4.1 Splitting Operation. The first one is simply a splitting or branching procedure. Let $S$ be a given subvariety in $\mathcal{S}$ with $K$ at position $\beta$. Then $S$ is the union of the two subvarieties $S^{\prime}$ and $S^{\prime \prime}$, where in position $\beta$ the $K$ is replaced by 0 in $S^{\prime}$ and by $M$ in $S^{\prime \prime}$. Thus $S^{\prime}$ is of smaller support than $S$, and in $S^{\prime \prime}$ we have a new position labeled with $M$ which allows for new applications of the elimination technique described next. In this situation we replace $S$ by $S^{\prime}$ and $S^{\prime \prime}$ on the stack.

4.4.2 Elimination Operation. This we refer to as an elimination or reduction operation. In its most elementary form it works as follows. Let $S$ be a string from the stack $\mathcal{S}$ with coefficient $M$ at $\beta$ and $K$ at $\alpha+\beta$, with $\alpha \in \Psi(P)$. Suppose that $S$ is invariant under the adjoint action of the root subgroup $U_{\alpha}$, that is $U_{\alpha} \cdot S \subseteq S$. Now let $x$ be an arbitrary element in the variety $S$. By definition, $x$ has a non-zero coefficient at $x_{\beta}$. By acting on $x$ with a suitable element from the root subgroup $U_{\alpha}$ of $P$ we can remove $\alpha+\beta$ from its support. Consequently, every $P$-orbit passing through $S$ also has a representative in the variety $S^{\prime}$ say, which (as a string) is obtained from $S$ by replacing the coefficient $K$ at $\alpha+\beta$ by 0 . By our assumption that $S$ is $U_{\alpha}$-invariant, no other roots are introduced into the support of $S^{\prime}$ in this process, and thus its support is smaller than that of $S$. Finally, we replace $S$ by $S^{\prime}$ on the stack $\mathcal{S}$. And we may repeat this elimination process with this new collection of strings.

MOP's elimination procedure is in fact considerably more intricate. Suppose now that in the example $S$ above a suitable operation with the root subgroup $U_{\alpha}$ removes the entry $K$ at $\alpha+\beta$, however, if there is a $K$, say at position $\gamma$ and a 0 at position $\alpha+\gamma$, then the action of $U_{\alpha}$ also produces a new entry in that coordinate. Nevertheless, suppose that this new entry can be removed again using a different root operation which in turn may or may not reintroduce new roots in the support of the resulting string. We can continue this procedure until no further new roots are being introduced. Then we have obtained a closed system of equations only involving the various positions and operators that are affected in this process. If the corresponding coefficient matrix is invertible, then the desired elimination can actually be performed. MOP checks whether such a system is solvable. The prime divisors which occur in such a process (in the Gaussian elimination) are written into a separate list in the record field factorlist of the output. Consequently, the result of the modality calculation is valid assuming that char $K$ is not in this factorlist. See the example of an output in Section 4.11.

In such a more involved elimination process new positions labeled with an " $M$ " or " $K$ " are introduced and a subsequent operator also acts on these new positions. Therefore, the order in which a set of operators is applied matters. In the course of analyzing a given string MOP simultaneously builds up all possible systems of equations aimed at eliminating a given targeted position in the support of the string respecting the different orders of operators. Once a consistent system of operators is obtained, MOP tries to eliminate the targeted position using this system. This guarantees that always a minimal set of operators is used.

Observe that the resulting system of equations is not linear in general. If the linear part of that system can be solved and if the resulting substitution from the solution of this linear part leads to a linear system on the remaining variables, then the complete system can be solved and the elimination can indeed be performed.

There are certain size and time restrictions built into MOP in order to limit the elimination process. If a critical time limit is reached MOP aborts its attempts to eliminate a certain element from the support of a string and proceeds to split at that targeted position. 
MOP eliminates a targeted position whenever possible. A splitting only takes place if all attempts to eliminate that position failed or if the attempt is timed out. As long as there are positions labeled with a " $K$ ", MOP proceeds to eliminate the one with the smallest root number, i.e., one of minimal height. Once all roots in $\operatorname{supp} S$ with coefficient " $K$ " have been removed, MOP proceeds to eliminate the positions labeled with " $M$ ".

The algorithm proceeds by an iteration and combination of these splitting and elimination operations. We have proven that $\bmod P$ is bounded above by $m$, once we have arrived at a stack $\mathcal{S}$ satisfying $\bmod (P: P \cdot S) \leq m$ for each $S$ in $\mathcal{S}$.

Observe that these two operations do in fact always yield a new stack, which is again labeled $\mathcal{S}$ for simplicity, that is another finite collection of strings with the desired property that every $P$-orbit through $\mathfrak{p}_{u}$ passes through some string in $\mathcal{S}$.

\subsection{Induction}

MOP works inductively in the following sense. Let $H$ be a proper semisimple regular subgroup of $G$ and let $Q=$ $P \cap H$. Inductively, $\bmod Q$ is known and in particular, we may assume that $\bmod Q$ is at most $m$, as otherwise $\bmod P>m$ by Lemma 2.3. It follows from the proof of Lemma 2.3 in [Röhrle 99, Prop. 2.2] that $\bmod (P$ : $\left.P \cdot \mathfrak{q}_{u}\right)=\bmod Q$. Therefore, we only need to consider the $P$-orbits in $\mathfrak{p}_{u} \backslash P \cdot \mathfrak{q}_{u}$. This applies to any such $Q$. Here it obviously suffices to only take those $H$ which are maximal among such subgroups leading to maximal candidates for $Q$. Hence, we only take maximal rank subgroups or Levi subgroups $H$ of corank 1 in $G$. We form the list of all conjugates of subsystems $\Psi(H)^{+}$, where $H$ runs through this fixed set of regular semisimple subgroups of $G$ of large rank, such that $\Psi(H)^{+} \subset \Psi^{+}$. We refer to this list of subsystems $\Psi(H)^{+}$as the InductionList of $G$.

If $G$ is of type $A_{r}$ or $D_{r}$, then we use Levi subsystems of type $A_{r-1}$ and $D_{r-1}$ with their usual embeddings. For $E_{6}, E_{7}$, and $E_{8}$ we also use maximal rank subsystems, e.g., for $E_{7}$ we use standard subsystems of type $E_{6}, A_{7}, A_{1} D_{6}$ and $A_{2} A_{5}$. The symmetric subsystems corresponding to such semisimple subgroups $H$ of $G$ are determined by means of the algorithm of Borelde Siebenthal, cf. [Bourbaki 75, Exc. Ch. VI §4.4]. In [Dynkin 57] all conjugacy classes of such subsystems of $\Psi$ under the action of the Weyl group of $G$ are classified; see also [Bala and Carter 76]. MOP computes the InductionList at each run anew.

For our purpose we need to examine the various parabolic subgroups $Q=P \cap H$ that actually occur in any given instance, where $\Psi\left(H^{+}\right)$runs through the InductionList of $G$ as defined above. MOP has a device to calculate each of these and writes the information into the output record with the record fields SubDiagrams and SubDiagramsDetail. In the first one MOP writes all occurring types of $Q$ and in the second lists explicitly all embeddings of $H$ into $G$ affording such $Q$ 's, see [Jürgens and Röhrle 01].

It is mandatory that we examine the list of subconfigurations in SubDiagrams, in order to ensure that no case is added to the induction list by mistake which does not satisfy $\bmod Q \leq m$, otherwise the outcome of the algorithm is meaningless.

Furthermore, this feature may help to find new cases of higher modality. For instance, the fact that $\bmod P>$ 0 in case $P=P_{J}$ in $E_{7}$ for $J=\left\{\alpha_{2}, \alpha_{3}, \alpha_{4}, \alpha_{5}\right\}$ was discovered by examining the information in this list of sub-configurations; here it turns out that one of the cases occurring is the Borel subgroup of a simple subgroup of type $A_{5}$, cf. [Jürgens and Röhrle 98, Lem. 3.13].

Because of the inductive nature of our method, and because of the fact that we simultaneously study a collection of orbits passing through strings, we do not get any information on the number of orbits in case we have shown that $\bmod P=0$.

\subsection{Managing the Stack}

If a sequence of splitting and elimination operations yields a string $S$ satisfying $\bmod (P: P \cdot S) \leq m$, then, instead of keeping it, we may simply delete $S$ from the stack $\mathcal{S}$. Thus, we will ultimately have reached our goal of showing that $\bmod P$ is at most $m$, precisely when all the strings which were generated in the course of this process have again been eliminated, that is when the resulting stack is empty. In our next section we discuss the various possibilities when a string $S$ can be removed from the stack, that is when $S$ satisfies $\bmod (P: P \cdot S) \leq m$.

The strings which we considered in the last section on elimination and splitting operations form a tree; we define $\mathfrak{p}_{u}$ to be the root of the tree, and for the elimination operation, $S$ is the parent node of $S^{\prime}$, and for the splitting operation, $S$ is the parent node of $S^{\prime}$ and $S^{\prime \prime}$. The leaves of this tree form a stack of the operation of $P$ on $\mathfrak{p}_{u}$. MOP starts with $\mathfrak{p}_{u}$ and builds up the tree until every leaf has at least one of the following two properties, either no splitting or elimination operation can be applied to it, or one of the criteria discussed below can be used to prove that its modality is at most $m$. In order to save memory and time, MOP does not keep the whole tree in the memory. Instead it enumerates the 
nodes of the tree in a depth-first-search order. Whenever it finds a string for which none of the criteria applies and none of the operations can be performed, MOP writes it into the CannotAnalyzeList. If this list is empty after a run of the program, then $\bmod P \leq m$, otherwise MOP computes an upper bound for the modality of $P$ from the strings in this list. The nodes of the tree are stored in a stack, which is the usual first-in-last-out data structure. The explicit use of a stack has the advantage that we can save it to a disc file and recover the data in case of a system crash. First MOP initializes the stack with a string which is just the string $\mathfrak{p}_{u}$ in the default setting. As long as there are strings left on the stack, MOP removes the one from the top. If one of the deletion criteria defined below applies to it, we have reached a leaf node and just continue. Otherwise we try to apply a splitting or elimination operation, put the resulting string(s) back onto the stack and continue. If these attempts fail, we have reached a leaf node again and apply the ExtendedOperation (see Section 4.8). If this operation does not prove that the string fulfills the modality condition, we add the string to the CannotAnalyzeList. In any case we continue with the next string on the stack.

\subsection{Deletion Criteria}

We now describe the four different deletion criteria which enable us to remove a string $S$ from the stack $\mathcal{S}$, that is criteria ensuring $\bmod (P: P \cdot S) \leq m$.

4.7.1 Redundancy Criterion. Instead of removing the string from the top of the stack immediately, we leave it there and mark it as "done". Whenever we find a string $S$ on the stack marked "done", we know that this string has been treated already. In this case MOP puts it into what is called the RedundancyList. Apart from merely writing $S$ into the RedundancyList, we also write every conjugate of $S$ under the simple reflections of the Weyl group $W_{P}$ of $P$ (i.e. those corresponding to $J$ ) into this list, as each such conjugate also can be considered as analyzed. In principle one could add the entire $W_{P^{-}}$ orbit of $S$ in $\mathfrak{p}_{u}$ to the RedundancyList. However, calculating the orbit is too time consuming. Initially, the RedundancyList is empty and in the course of the program run MOP adds strings to it which have already been analyzed. Thus, compared to the induction list, this is a dynamic list which is updated continually. Whenever we find a string $S$ which is a subvariety of a string in the Redundancylist, we may consider $S$ also as analyzed and in this case we simply drop $S$.
Before a string $S$ is added to the RedundancyList we first compare $S$ with any string $S^{\prime}$ already in the RedundancyList. If $S$ is a subvariety of some $S^{\prime}$ on this list, then we do not add $S$. On the other hand, any such $S^{\prime}$ which is itself a subvariety of $S$ is removed from the RedundancyList and $S$ is added instead. This comparison feature guarantees that we maintain an optimal RedundancyList at any time.

4.7.2 Induction Criterion. Suppose that in the course of our analysis we encounter a string $S$ which satisfies supp $S \subseteq \Psi(H)^{+}$, where $\Psi(H)^{+}$is a member of the InductionList of $G$, that is $S \subseteq \mathfrak{p}_{u} \cap \mathfrak{h}=\mathfrak{q}_{u}$, where $Q=P \cap H$. Then, by induction (cf. Section 4.5), we infer that $\bmod (P: P \cdot S)=\bmod (Q: Q \cdot S) \leq \bmod Q \leq m$, and thus we can remove $S$ from $\mathcal{S}$. The equality part of this statement follows from the proof of Lemma 2.3 in [Röhrle 99]. We refer to this as the induction criterion.

4.7.3 Rank Criterion. Another situation when we can delete a string $S$ from the stack, arises in the following way. Suppose that the support of $S$ consists of at most $\operatorname{dim} T+m$ roots. Let $d$ be the number of linearly independent roots in $\operatorname{supp} S$. Note that $d$ is at most $\operatorname{dim} T$. Then for an arbitrary element $x$ in $S$ we can apply suitable elements from $T$ to scale as many as $d$ coefficients of $x$ to equal 1 with at most $|\operatorname{supp} S|-d$ coefficients of $x$ remaining free. Now, if $|\operatorname{supp} S|-d \leq m$, then the resulting set of $P$-orbits passing through all the elements which are obtained by varying the entries in the remaining free coefficients depends on at most $m$ parameters, and thus, $\bmod (P: P \cdot S)$ is bounded above by $m$, as desired. Thus, we can remove $S$ from the stack $\mathcal{S}$. If $|\operatorname{supp} S| \leq m+d$, then we say that $S$ satisfies the rank criterion.

4.7.4 J-height Criterion. Finally, we have one further possibility to eliminate strings from the stack. Suppose that for $S$ in $\mathcal{S}$ each root in the support of $S$ has a fixed $J$-height $l \in \mathbb{N}$. Since MOP only applies when $G$ is simply laced, the hypothesis of Remark 2.5 on char $K$ is fulfilled. Thus we have $S \subseteq \oplus \mathfrak{g}_{\beta}\left(\cong \mathfrak{p}_{u}^{(l-1)} / \mathfrak{p}_{u}^{(l)}\right)$, where $\beta$ runs through all roots in $\Psi$ of $J$-height $l$. Now by Lemma 2.6 there are only finitely many orbits of the standard Levi subgroup of $P$ on this space; whence there are only finitely many $P$ orbits passing through $S$ and thus we can remove $S$ from the stack; then we say that $S$ satisfies the $J$-height criterion.

4.7.5 Effectiveness of the Criteria. In the course of a run of MOP, each of these criteria may occur many times. However, the induction and the redundancy criteria are 
generally the more effective ones of the four. Their advantage is twofold over the others. Firstly, they only involve a subset check of the support of the string at hand and the members of the induction list of $P$, or of the redundancy list. In terms of computing time this is not too costly provided both of these lists are short. Secondly and more importantly, these criteria allow us to remove strings from the stack which may have large support. Else these might take a long time to be analyzed after being broken down into smaller strings using the elimination and branching operations. The fact that we add all conjugates of any analyzed string under the generators of the relative Weyl group $W_{P}$ to the RedundancyList (cf. 4.7.1) makes this a very effective criterion. On the other hand, the rank criterion can only be applied when $\operatorname{supp} S \leq \operatorname{dim} T+m$ which is usually small compared to $\operatorname{dim} \mathfrak{p}_{u}$. A further disadvantage of it is that it requires calculating the rank of matrices which is less favorable in terms of computing time than a simple subset check as involved in the other three criteria.

\subsection{ExtendedOperation}

In the course of applying splitting and elimination operations to strings from the stack $\mathcal{S}$ it rarely happens that in the end all the strings that are produced in this fashion satisfy one of the four deletion criteria from above. Ultimately, it may happen that strings $S$ occur in $\mathcal{S}$ which have the property that the coefficient of any root in $\operatorname{supp} S$ is $M$ (that is no further branching operations are possible), no further elimination is possible (or aborted due to time limitations), and $S$ does not satisfy any of the four deletion criteria above, so it cannot be removed from the stack. Then MOP enters a process to which we refer to as ExtendedOperation. We choose a total ordering of all possible operators, that is of $\Psi(P)$, starting with the negative roots, ordering by height. We apply these operators consecutively to $S$ and produce entirely new strings with new entries in that fashion. The resulting strings are then analyzed further with the aforementioned splitting and elimination techniques. That is we return to the usual procedure with these new strings created in the ExtendedOperation. This is a systematic way to obtain a large number of new admissible strings that can be analyzed further.

\subsection{Prime Restrictions}

In terms of restrictions on char $K$, MOP's results have to be interpreted as follows. If MOP is run using the InductionList of $G$ and $G$ is of exceptional type, then the results obtained are only valid provided char $K$ is not a bad prime for $G$, cf. Lemma 2.3. If $G$ is of classical type, then using this list does not imply any characteristic restrictions, as here only Levi subsystems are involved in the construction, cf. Remark 2.4. MOP allows a user to disable this inductive feature, e.g., see the example in Section 4.11. Results obtained without using the InductionList are valid subject only to characteristic restrictions stemming from the factorlist of the output record, see 4.4.2. Often a certain bad prime $p$ does not occur in that list, and thus, the modality statement computed is also valid in case char $K=p$. Such an example is illustrated in Section 4.11. This shows that certain instances of Theorem 3.1 may also be valid in specified bad characteristics as well.

\subsection{Counters}

MOP keeps track of a number of parameters. It counts the number of strings that are analyzed during a run of the algorithm. Each time a string is taken off the stack we raise a counter by one. Another counter keeps track of the number of splitting operations that are performed. Of particular interest is the success of the various deletion criteria; there is a counter for each of the four deletion criteria. Apart from these MOP also has a counter for the number of calls of the internal function ExtendedOperation, cf. 4.8; see [Jürgens and Röhrle 01]. If there is no call of ExtendedOperation, then the final values of the counters for the various deletion criteria and the splitting operation add up to the value of the counter for the strings. Viewing the strings created by MOP in form of a tree as indicated in 4.6 , this amounts to counting all leaf nodes (deletion criteria) together with all internal branching nodes (splittings). If there are calls of ExtendedOperation, then the sum of the final values of these counters can exceed the counter for the strings, as here new branchings may happen before MOP returns to the stack. This, for instance, happens in Example 4.11.

\subsection{An Example}

We illustrate a call of MOP in the $E_{8}$ instance when $J=$ $\Pi \backslash\left\{\alpha_{7}\right\}$ with the use of the induction list disabled.

gap> RequirePackage("mop");

gap> $r:=$ Modality ("E" , 8, $[1,2,3,4,5,6,8]$, rec(UseInductionList : $=$ false));

The first line reads the GAP package MOP initializing a record MOP and defining a function Modality. This function returns a record with the results of the computation written in its record fields. All the other functions are internal and are therefore located in the record 
MOP. The first three parameters of Modality are mandatory; they define the type of $G, \operatorname{rank} G$, and the subset $J$ of simple roots defining $P$. The fourth parameter is optional; it allows to overwrite the default setting for a number of global parameters. In our example we set UseInductionList: $=\mathrm{false}$ in order to suppress the use of the induction list. For a detailed list of all optional parameters, we refer to the MOP manual [Jürgens and Röhrle 01].

After a run of MOP the results can be displayed by the print command in GAP for records:

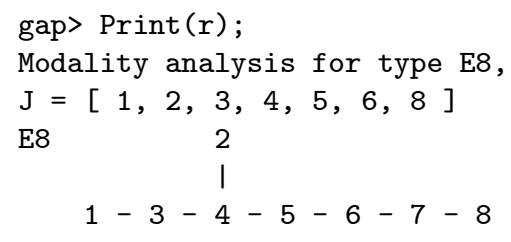

0 induction list matches

588 already done

201 occurrences of rank condition

$112 \mathrm{~J}$-Height criterion invoked

1 extended operations

0 unresolved strings

characteristic restrictions: [ 2, 3 ]

The modality of $\mathrm{P}$ is 0 .

The display is more or less self-evident. Apart from the case studied, MOP prints the result of the counters, the characteristic restrictions stemming from solving various systems of equations, as well as the modality calculation. Observe that the prime 5 does not occur in the factorlist and consequently, this finiteness result is also valid in characteristic 5, cf. Section 4.9.

\subsection{Safety Feature}

In order to recover intermediate results already obtained in case of a system crash, we periodically save all this information and the status of the analysis to a file with the suffix . save depending on $J$; e.g., E8_1_2_3_4_5_6_8.save. If such a file exists and the function Modality is called again with the same parameters, then MOP reads the information and status from this external file and proceeds the analysis. The default setting for the time period after which this data is recorded anew is 20 minutes.

\subsection{The Verbose Feature}

By setting an optional parameter when calling MOP in the function Modality the algorithm prints out various pieces of information of the entire analysis. In principle, this allows a user to check every detail of MOP's calculation by inspection. For details of this feature and an example in the verbose mode, see the manual [Jürgens and Röhrle 01].

\section{ACKNOWLEDGMENTS}

We are grateful to G. Hiss and F. Lübeck for helpful suggestions concerning programming in GAP.

\section{REFERENCES}

[Azad et al. 90] H. Azad, M. Barry, and G. Seitz, "On the structure of parabolic subgroups," Com. in Algebra 18 (1990), 551-562.

[Bala and Carter 76] P. Bala and R.W. Carter. "Classes of unipotent elements in simple algebraic groups. II," Math. Proc. Cambridge Phil. Soc., 80 (1976), 1-18.

[Borel 91] A. Borel. Linear Algebraic Groups, Graduate Textbooks in Mathematics, 126, Springer Verlag, New YorkBerlin, (1991).

[Bourbaki 75] N. Bourbaki. Groupes et algèbres de Lie, Chapitres 4,5 et 6, Hermann, Paris, 1975.

[Brüstle and Hille 00] T. Brüstle and L. Hille. "Finite, Tame and Wild Actions of Parabolic Subgroups in $G L(V)$ on Certain Unipotent Subgroups." J. Algebra, 226 (2000), 347-360.

[Bürgstein and Hesselink 87] H. Bürgstein and W.H. Hesselink. "Algorithmic orbit classification for some Borel group actions." Comp. Math., 61 (1987), 3-41.

[Dynkin 57] E.B. Dynkin. "Semisimple subalgebras of semisimple Lie algebras." Amer. Math. Soc. Transl. Ser. 2, 6 (1957), 111-244.

[GAP 97] The GAP Group, Lehrstuhl D für Mathematik, RWTH Aachen, Germany and School of Mathematical and Computational Sciences, U. St. Andrews, Scotland. GAP-Groups, Algorithms, and Programming, Version 3.4.4, 1997

[Geck et al. 96] M. Geck, G. Hiss, F. Lübeck, G. Malle and G. Pfeiffer. "CHEVIE - A system for computing and process ing generic character tables for finite groups of Lie type, Weyl groups and Hecke algebras." AAECC, 7 (1996), 175-210.

[Hille and Röhrle 99] L. Hille and G. Röhrle. "A classification of parabolic subgroups of classical groups with a finite number of orbits on the unipotent radical." Transformation Groups, 4:1 (1999), 35-52. 
[Jürgens and Röhrle 98] U. Jürgens and G. Röhrle. "Algorithmic Modality Analysis for Parabolic Groups," Geom. Dedicata, 73 (1998), 317-337.

[Jürgens and Röhrle 01] U. Jürgens and G. Röhrle. The MOP Manual, Manuscript (2001).

[Kashin 90] V.V. Kashin. "Orbits of adjoint and coadjoint actions of Borel subgroups of semisimple algebraic groups." (Russian), Problems in Group Theory and Homological algebra, Yaroslavl'. (1990), 141-159.

[Popov 97] V.L. Popov. "A finiteness theorem for parabolic subgroups of fixed modality." Indag. Math. N. S. :1 (1997), 125-132.

[Popov and Röhrle] V.L. Popov and G. Röhrle. "On the number of orbits of a parabolic subgroup on its unipotent radical" In Algebraic Groups and Lie Groups, Australian Mathematical Society Lecture Series 9, Editor G. I. Lehrer, pp. 297-320, Cambridge University Press, Cambridge, UK (1997).
[Popov and Vinberg 94] V.L. Popov and E.B. Vinberg. "Invariant Theory." In Encyclopaedia of Math. Sci.: Algebraic Geometry IV. Springer-Verlag, 55, (1994), pp. 123284, Berlin, Springer-Verlag. (Translated from Russian Series: Itogi Nauki i Tekhniki, Sovr. Probl. Mathem., Fund. Napravl. 55 (1989), 137-314).

[Richardson 74] R.W. Richardson. "Conjugacy classes in parabolic subgroups of semisimple algebraic groups." Bull. London Math. Soc. 6 (1974), 21-24.

[Richardson 85] R.W. Richardson. "Finiteness Theorems for Orbits of Algebraic Groups." Indag. Math. 88 (1985), $337-344$.

[Röhrle 96] G. Röhrle. "Parabolic subgroups of positive modality." Geom. Dedicata 60 (1996), 163-186.

[Röhrle 97] G. Röhrle. "A note on the modality of parabolic subgroups." Indag. Math. N.S. 8:4 (1997), 549-559.

[Röhrle 99] G. Röhrle. "On the modality of parabolic subgroups of linear algebraic groups." Manuscripta Math. 98 (1999), 9-20.

Ulf Jürgens, Fakultät für Mathematik, Universität Bielefeld, 33615 Bielefeld, Germany (ulf@mathematik.uni-bielefeld.de)

Gerhard Röhrle, School of Mathematics and Statistics, The University of Birmingham, Edgbaston, Birmingham B15 2TT, United Kingdom (ger@for.mat.bham.ac.uk)

Received January 30, 2001; accepted in revised form January 11, 2002. 